\title{
Brahms, el romanticismo y el desarrollo potencial de la viola
}

\section{Brahms, romanticism and the potential development of the viola}

\author{
JUAN-CARVAJAL, Mara Lioba*†, VDOVINA, María y SÁNCHEZ-USÓN, María José
}

Universidad Autónoma de Zacatecas

ID $1^{\text {er }}$ Autor: Mara Lioba, Juan-Carvajal / ORC ID: 0000-0001-6968-3813, Researcher ID Thomson: P-7756-2016, CVU CONACYT ID: 216443

ID 1 er Coautor: María, Vdovina / ORC ID: 0000-0001-6656-0789, Researcher ID Thomson: S-7917-2018

ID $2{ }^{\text {er }}$ Coautor: María José, Sánchez-Usón / ORC ID: 0000-0002-3409-4055, Researcher ID Thomson: S-7908-2018, CVU CONACYT ID: 432522

DOI: $10.35429 /$ JPDL.2020.19.6.7.20

Recibido 29 de Septiembre 2020; Aceptado 30 Diciembre, 2020

\section{Resumen}

La viola ha jugado un papel diferente respecto al violín en el desarrollo de los cordófonos, ello ha impactado también en su reconocimiento social; no obstante, su potencialidad sonora y expresiva se ha ido evidenciando a través de los siglos. Con la aparición de diferentes estilos artísticos, los compositores han utilizado el instrumento de manera creadora, ampliando sus capacidades técnico-expresivas, lo que propició un mayor nivel de preparación en los intérpretes y su habilidad en el dominio de los repertorios musicales. Ellos no sólo acogen las nuevas obras, sino que, a su vez, proyectan nuevos retos. Este trabajo tiene como objetivo reconocer el papel del romanticismo como movimiento estético en el impulso al amplio crecimiento compositivo e interpretativo de la viola, a partir de algunas composiciones para la música de cámara de Johannes Brahms. Como estrategia metodológica se combinan la revisión historiográfica, el análisis contextual y el análisis de documentos, con lo que se destaca el lugar de la viola en el conjunto instrumental, su rol protagonista y las composiciones brahmsianas para este instrumento musical.

Johannes Brahms, La música de cámara romántica, La viola

\begin{abstract}
The viola has played a different role from the violin in the development of chordophones, this has also impacted on its social recognition; however, its sonorous and expressive potential has been evident throughout the centuries. With the appearance of different artistic styles, composers have been used the instrument in a creative way, expanding their technicalexpressive capacities, which has favored a higher level of preparation in the interpreters and their ability in the mastery of musical repertoires. They not only welcome new works, but also project new challenges. This work aims to recognize the role of romanticism as an aesthetic movement in the promotion of the broad compositional and interpretative growth of the viola, from some compositions for the chamber music of Johannes Brahms. As a methodological strategy, historiographic review, contextual analysis, and document analysis are combined, highlighting the place of the viola in the instrumental ensemble, its leading role and the Brahms compositions for this musical instrument.
\end{abstract}

Johannes Brahms, The romantic chamber music, The viola

Citación: JUAN-CARVAJAL, Mara Lioba, VDOVINA, María y SÁNCHEZ-USÓN, María José. Brahms, el romanticismo y el desarrollo potencial de la viola. Revista de Filosofía y Cotidianidad. 2020, 6-19: 7-20

\footnotetext{
* Correspondencia del Autor (maralioba@ hotmail.com)

$\dagger$ Investigador contribuyendo como primer Autor.
} 


\section{Introducción}

Desde el barroco musical y hasta la actualidad, el desarrollo de la música ha ido aparejado al de los instrumentos que en ocasiones se perfeccionan, o se transforman, para responder a las necesidades estéticas y expresivas de cada época. La viola, como el instrumento actual, surge con el nacimiento de la familia del violín alrededor del siglo XVI y durante el XVII. Se caracteriza por ser un instrumento con ciertas peculiaridades constructivas que distinguen la relación entre su tamaño y la acústica sonora, lo que se evidencia tanto en las variantes de su diseño físico y estructural como en la calidad y diversidad tímbrico-sonora resultante. Su sonido suave, profundo, cálido e íntimamente humano ha sido identificado por muchos compositores que lo han explorado exponencialmente en sus creaciones.

En cada movimiento artístico existieron compositores que han prestado atención a la viola y la han apreciado de manera diferente, impulsando la evolución del procedimiento compositivo y diversificando el papel desempeñado dentro de las agrupaciones instrumentales.

En la historia de la música hay pasajes poco difundidos, como, por ejemplo, el hecho de que uno de los más altos exponentes del barroco alemán, el compositor J. S. Bach (1685-1750), entre los muchos aportes que realizara en su vida artística estuviera el de componer para la viola y tocarla en los conjuntos. En el set de instrumentos de cuerdas que construían los luthiers o lauderos famosos de la época se incluían, al menos, dos violas (no hay que olvidar que en ese entonces todavía estaban en uso y franca coexistencia, además de la familia del violín, la antigua familia de las violas $d a$ gamba y da braccio, y que los roles interpretativos, fueron pasando equitativamente de unos instrumentos a otros). Bach compone para varios singulares conjuntos instrumentales los Six concerts avec plusieurs instruments (Seis conciertos de Brandeburgo BWV 1046-1051); de ellos el Concerto 6to à due Viole da braccio, due Viole da Gamba, Violoncello, Violone $e$ Cembalo (Sexto concierto de Brandeburgo) está escrito para un conjunto instrumental sin violines, llevando el rol principal dos violas "solistas" en un estrecho canon, dejando el descanso armónico en el resto de los instrumentos.
Según B. Dobrojotov, Bach disfrutaba tocar la parte de la primera viola: En Berlín Bach tocó para Christian Ludwig y le regaló una de sus obras interpretadas que se supone fue el sexto concierto (Dobrojotov, 1962, pp. 9 y 10).

La viola se inserta también en el rol de los conjuntos, y como solista durante el preclásico y el clásico de finales del siglo XVII y todo el XVIII de manera diferente, ya que, en este lapso surgen nuevos géneros instrumentales y se desarrollan otros preexistentes (por un lado, está la línea del concierto sobre la base del estilo concertante y solista, incluyendo la orquesta y el género sinfónico, y por otro, la música de cámara, principalmente la sonata y el cuarteto).

En ese tiempo, los intérpretes violinistas apreciaron tocar la viola; destacaron de manera particular los de la Escuela de Mannheim, donde los compositores, intérpretes y directores de orquesta asistentes en Bohemia, Bélgica, Austria e Italia, además de diversas regiones de la actual Alemania, se dieron cita, permitiendo la propagación de la música instrumental y el desarrollo de la sinfonía concertante, tanto en instrumentos de cuerdas como de vientos. También, Franz Joseph Haydn (1732-1809), considerado el padre del cuarteto de cuerdas, estableció el cuarteto clásico integrado por dos violines, una viola y un violonchelo, y con ello, asignó un lugar a la viola, tanto como integrante estable del conjunto (a diferencia de otros tipos de ensambles barrocos), como en la función de su propia voz. También, prolifera la composición de dúos para violín y viola, y aunque esta todavía no tiene un papel protagónico en su expresión y complejidad técnica, su instalación dentro de los géneros principales del clasicismo le cimienta su proyección futura.

De manera particular, sobresale la figura de W. A. Mozart (1756-1791), quien presta atención al tratamiento de la viola en los conjuntos de cámara, como solista o en la orquesta. Ejemplo de ello es el Trío para clarinete, viola y piano en Mib Mayor K 498 (1786), conocido como Kegelstatt-trío. Esta experiencia de combinar la viola y el clarinete (instrumento entonces de reciente creación) fue replicada posteriormente por algunos románticos. 
La técnica de composición respecto a la independencia de la viola, y su significado como voz intermedia, cambió tanto en los ensambles de cámara que incluía los cuartetos y quintetos (se sabe que Mozart trabajó en trece quintetos para dos violas, y de ellos, descartó siete) como en el género sinfónico (Guibert, 2003, p. 12). Su obra culminante en ese sentido, dado el papel igualitario otorgado a la viola y al violín incluyendo su cadencia, fue la Sinfonía Concertante en Mib Mayor para violín, viola y orquesta, K. 364 (1779), donde, además de la viola solista, se incluyen dos partes de viola dentro de la orquesta.

Hacia el romanticismo, la viola se adopta como un instrumento ideal para la expresión interior; si bien en el afianzamiento del género de concierto había logrado cierta relevancia, contrariamente, ahora la mirada está en el dibujo, en la paleta de colores y timbres que provocan sentimientos que tocan la sensibilidad humana, por lo que destacará de manera distintiva en los diversos ensambles y, aún como solista, bajo esta mirada. La música de cámara alcanza un importante desarrollo, partiendo de las variantes clásicas del cuarteto de cuerdas impulsado a mediados del siglo XVIII por P. Nardini (17221793), C. D. von Dittersdorf (1739-1799), L. Boccherini (1743-1805), J. Haydn, y L. van Beethoven (1770-1827), a quien se enmarca como el músico que cierra la época del clasicismo vienés y abre el camino hacia el romanticismo.

En el cuarteto, durante este periodo, destacan también F. Schubert (1797-1828), R. Schumann (1810-1856), A. Dvorák (18411904), B. Smetana (1824-1884) y P. I. Tchaikovsky (1840-1893). Por otro lado, algunos compositores se ocuparon de continuar desarrollando la viola solista, tal fue el caso, por ejemplo, del violinista, violista y compositor italiano A. Rolla (1757-1841) y de su alumno N. Paganini (1782-1840), quien compuso la Sonata para la Gran Viola y orquesta, e inspiró la creación de Haroldo en Italia Op. 16, una especie de sinfonía con una viola solista del compositor francés H. Berlioz (1803-1869).
Son muchos los románticos que trabajan prestando atención a la sonoridad de la viola; además de los mencionados vale destacar a $\mathrm{F}$. Mendelssohn (1809-1847), M. Glinka (18041857) y M. Bruch (1838-1920), quien compuso el Concierto para clarinete y viola Op. 88 y las Ocho piezas para clarinete, viola y piano, en las que la viola resalta, primeramente, por su color y expresividad.

A tenor de lo anteriormente expuesto, hay que prestar atención al papel de la viola en el trabajo camerístico del compositor alemán nacido en Hamburgo en 1833 y posteriormente residente en Viena hasta su fallecimiento en 1897: Johannes Brahms.

Brahms, el niño prodigio, pianista $\mathrm{y}$ compositor, comenzó los estudios musicales con su padre; posteriormente estudió piano con O. F. W. Cossel (1813-1865) y composición con E. Marxsen (1806-1887). En 1853 compartió una gira como intérprete del piano con el violinista húngaro E. Reményi, quien lo introdujo con el famoso violinista Josef Joachim (1831-1907), que se convertiría en su amigo, además de consultor y crítico de su obra, y a quien dedicó, entre otras, su Concierto para violín op. 77 en Re mayor.

A través de éste, Brahms conoce también a Schumann: “...ya se sabe que Robert Schumann iba a ver en Brahms a un «nuevo mesías del arte», así como [de] la apasionada amistad que se estableció entre Johannes y Clara, que perduró hasta la muerte de ésta. El año de 1853 -decididamente fastuoso- terminaría con una estancia triunfal en Leipzig, donde recibió ánimos de Berlioz y de Liszt." (Tranchefort, 2005 , p. 247). Un año más tarde, en 1854 , comienza la carrera pública del Brahms compositor, con su Trío para piano y cuerdas en Si mayor $O p$. 8, que culminaría dos años antes de su muerte.

Este trabajo tiene como objetivo reconocer el papel del romanticismo como movimiento estético en el impulso al crecimiento compositivo e interpretativo de la viola, a partir de algunas composiciones para la música de cámara de Johannes Brahms. 


\section{Metodología}

En este artículo, varias son las estrategias metodológicas empleadas para la consecución del objetivo propuesto. Obligadamente, se parte de una revisión historiográfica de la viola en la cronología decimonónica, destacando sus singulares, ubicándola en el conjunto instrumental existente y enfatizando su imparable proceso de individualización solística. El análisis contextual de Brahms es asimismo considerado, incardinando en él no sólo pormenores de su vida y obra, sino también reflexiones claves y críticas del momento sociocultural que vivió en una Alemania generadora del poderoso movimiento romántico. Finalmente, una revisión y descripción de las composiciones brahmsianas para viola, conocidas desde la teoría musical y desde la práctica de la interpretación, ejemplifica el rol protagonista jugado por este instrumento cordófono en el siglo XIX.

\section{Desarrollo}

\section{El romanticismo: más que un movimiento artístico}

Tradicionalmente, se ha vinculado siempre el término "romanticismo" al adjetivo "romántico", como si el segundo se inscribiera en una idea temporal, caracterizando todas y cada una de sus expresiones y a sus respectivos protagonistas. En su libro Romanticismo. Una odisea del espíritu alemán, el filósofo alemán Rüdiger Safranski (2009) precisa y matiza esta relación al afirmar que "romanticismo" se refiere a una época y "romántico" a una tendencia o actitud que no se limita únicamente a un tiempo en concreto, sino que puede prolongarse más allá de unos límites cronológicos, incluso surgir de una manera precursora en años precedentes a los enmarcados por las últimas décadas del siglo XVIII y el siglo XIX. Partiendo de esta opinión, no extraña, pues, que el vocablo "romántico" se usase ya en el siglo XVII, pero más como una voz despectiva que como un calificativo de observación positiva, aplicada a un tipo de literatura "barata", "romanesca", carente de valor y mérito literario, si bien tremendamente popular. Esta connotación de falta de refinamiento y aun de vulgaridad será uno de los atractivos del romanticismo, convirtiéndose en un bien cultural, al rescatar y poner en valor aspectos que la Ilustración había relegado, como lo religioso, lo pintoresco o lo medieval.
Es útil, además, hacer notar de entrada que "nuestros románticos" no se llamaron a sí mismos románticos, sino la "nueva escuela". Esta denominación la usó el propio Friedrich Schlegel cuando preparaba la edición de sus Obras, y, en particular, cuando preparaba la reedición de su reconocido escrito de 1800, Diálogo sobre la poesía, que puede considerarse el documento que compendia el significado de la sensibilidad y el gusto nuevo del romanticismo, frente al espíritu dieciochesco, de tradición racionalista y de gusto clasicista [...] Para "nuestros románticos" los románticos no eran ellos, ellos se sentían infortunadamente "modernos", sino que los románticos por excelencia eran Dante, Ariosto, Tasso, Cervantes y, sobre todo, Shakespeare. (Domínguez, 2009, p. 48)

Asumiendo las anteriores precisiones, este pujante movimiento de amplio alcance, originado en Alemania, Inglaterra y Francia, y extendido por la totalidad del continente europeo, principalmente en el siglo ochocentista, comportó la imposición de nuevos principios opuestos a la rigidez del racionalismo ilustrado, que permearon en todas las manifestaciones del arte, tales como las pasiones exacerbadas, la emoción, la introspección, el culto al ego, la valoración del cristianismo, lo espiritual y lo sobrenatural, el hallazgo de la inspiración en tiempos, tierras y culturas lejanas y exóticas, la insatisfacción vital, la melancolía, la vivencia del amor no correspondido, etc. En suma: un conjunto de ideas que conformarán una diferente mentalidad individual y colectiva, fundada, en gran medida, por el arte.

Lo que ahora suscitan en nosotros las obras de arte [afirmaría Hegel] es, además del goce inmediato, también nuestro juicio, pues lo que sometemos a nuestra consideración pensante es el contenido, los medios de representación de la obra de arte y la adecuación o inadecuación entre ambos aspectos. (Hegel, 1989, p. 14)

De esta forma, la creación se impone, de un modo revolucionario no considerado hasta entonces frente a cánones establecidos de equilibrio, proporción y racionalidad universales. 
Es así que los nuevos valores antes enumerados impregnaron las vidas y las obras de los artistas románticos, de los cuales, desde un planteamiento filosófico, bien pudiera decirse que se caracterizaron por un profundo anhelo metafísico y la ausencia de un principio de realidad (Domínguez, 2009), que les impulsaba a huir de lo utilitario, lo práctico y convencional. Paradójicamente, esta huida de la materialidad, obraría en su favor, ya que los creadores adquirirán un estatus social más reconocido y, sobre todo, una mayor independencia creativa.

En síntesis, el romanticismo supuso una verdadera revolución ideológica, que afectaría a la generalidad de los órdenes artísticos. La música no sería una excepción. Sus cultores se verían libres de traspasar los límites de lo meramente compositivo para poder desempeñarse, de igual manera, como intérpretes, directores de orquesta o escritores. También, volverían sus ojos al ámbito de la intimidad musical, aunque, y en sorprendente contraste, se asistirá a la exhibición del virtuosismo interpretativo, un lenguaje musical más complejo y una notoria evolución y amplitud orquestal. Todavía, el peso de la tradición precedente es fuerte; empero en ella se incardinan los cambios que, paulatinamente actuarán como cuñas estéticas que fragmentan la rígida estructura musical anterior.

De cette manière générale, le romantisme adopte tous les genres de l'époque classique en les transformant et adaptant, mais en invente de nouveaux: pièce brève pour piano, le lied, le poème symphonique, le drame musical. Sur le plan du langage musical, les compositeurs développent l'harmonie (allant parfois aux limites de l'atonalité par l'usage de chromatismes, altérations ou enharmonies), la mélodie (qui doit peindre les sentiments), le rythme (superposition de duolets et triolets par exemple) et se sent très attirés par des timbres instrumentaux proches de la nature (cor de chasse, flûte, clarinette). (Ars classical, 2019, s./p. $)^{l}$

\footnotetext{
1 "En general, el romanticismo adopta todos los géneros de la época clásica, transformándolos y adaptándolos, pero inventa nuevos: la pieza breve para piano, el lied, el poema sinfónico, el drama musical. En cuanto al lenguaje musical, los compositores desarrollan la armonía (a veces llegando a los límites de la atonalidad por el uso de cromatismos, alteraciones o enarmonías), la melodía (que debe dibujar los sentimientos), el ritmo (superposición de dúos y tríos, por ejemplo) y se sienten muy atraídos por los timbres instrumentales cercanos a la naturaleza (cuerno de caza, flauta, clarinete)".
}

ISSN: 2414-8857

ECORFAN® Todos los derechos reservados
En esta complicada balanza entre el pasado y la modernidad se situará Brahms, señalado por unos como un creador netamente neo-clasicista (Le Diagon-Jacquin) y por otros como un progresista (Schoenberg). Sin embargo, este contrarresto, difícil de mantener, se alteró, principalmente a partir de 1850 , inicio de la última etapa del romanticismo o romanticismo tardío, en la que el compositor hamburgués se posicionó en "la guerra de los románticos", en contra de la música programática defendida por los músicos de la corriente de Weimar, representada por R. Wagner (1813-1883) y F. Liszt (1811-1886). Frente a éstos, que preconizaban el contenido y la expresión de los sentimientos en la música, Brahms apoyó un ideal estético apegado al clasicismo de las formas musicales y de la armonía, defendiendo la música de cámara, y en ella la sonata y el sexteto. No obstante, pese a su ubicación conservadora, Brahms renueva el trabajo compositivo dotando a las antiguas formas y estilos musicales de una dimensión propia, caracterizada por la inimitable belleza de sus sonoridades.

\section{El romanticismo y la estética brahmsiana}

El musicólogo estadounidense León Plantinga (2015), especialista en la música europea del llamado período romántico, explica en sus estudios cómo las circunstancias imponen al músico decimonónico una visión diferente de su actividad tradicional bajo el mecenazgo, al figurar la música entre las llamadas "Bellas Artes" y enfrentar el éxito o el fracaso ante el gran público en las salas de conciertos o en los teatros. Expone, también, la influencia que en este terreno tuvo el historicismo, lo que llevó a la "recuperación histórica de estilos antiguos", ello incluyó el reconocimiento de la figura de Bach, olvidado durante un largo período de la historia musical, y la definición de los clásicos a través de las figuras de Haydn, Mozart y Beethoven. 
La recuperación y su injerencia en las corrientes musicales del momento a comienzos del siglo XIX tuvo una serie de consecuencias cruciales para el compositor romántico. En primer lugar, se enfrentaba a un desafio sin precedentes: sus obras estaban obligadas a competir no solamente con las de sus contemporáneos, sino también con los grandes maestros de las generaciones precedentes. Cuando Schubert, Schumann y Brahms expresan su desánimo ante la perspectiva de tener que componer sinfonías y cuartetos tras la sombra de Beethoven no les faltaba razón: las sinfonías de Beethoven aún se escuchaban en las salas de concierto de toda Europa, estableciendo un listón muy alto a partir del cual se juzgaba la obra del resto de los compositores. [...] Los géneros tradicionales de música instrumental no fueron cultivados sistemáticamente por los compositores jóvenes en las primeras décadas del siglo XIX. Las sinfonías, las sonatas y los conciertos fueron desplazados en gran medida por una intensa proliferación de obras de pequeñas dimensiones $y$ de variada procedencia: música relacionada con la danza, fantasías, baladas, intermezzos y similares. La mayor parte de los primeros románticos tardaron mucho tiempo en familiarizarse con las grandes formas tradicionales: y las formas que se habían convertido en símbolo de la tradición parecían ser patrimonio de aquellos compositores que las habían convertido en lo que eran. (Plantinga, 2015, pp. 29 y 30)

No hay dudas de que Johannes Brahms fue uno de los compositores románticos que enfrentaron esa ambigua realidad. Cabe preguntarse si el Brahms provinciano, pianista y compositor, con una estricta formación y respeto a lo clásico, conocedor de las tradiciones nacionales y del folclor húngaro, no padeció la misma incertidumbre sobre sí mismo que han sostenido por mucho tiempo los críticos acerca de cómo "clasificarlo" estilísticamente.

Si bien hubo de manifestarse contra la tendencia de la Nueva Escuela Alemana (1860), también fue muy creativo e ingenioso en el tratamiento de las voces interiores de sus creaciones, en el concepto unívoco y el acabado formal, y compuso obras y movimientos de ellas inspirado en el folclor y en las tradiciones.
Lo que, por un lado, puede definir a Brahms como el pináculo de la tradición clásica y academicista desde el uso de las formas es, a su vez, lo que lo convierte en un innovador de la expresión romántica, en cuanto al tratamiento de las ideas y motivaciones musicales, así como las fuentes de inspiración. En un análisis más interior del contenido íntimo de sus partituras, se ha demostrado cómo su estilo propio lo lleva a ser, también, un representante progresista ante lo moderno.

De no haber llevado Brahms el peso del historicismo romántico, de no haber sido tan riguroso consigo mismo y con su obra, de no haber deshecho tanto material (sobre todo en su etapa inicial), tanta partitura e ideas, de no haber sido fuertemente criticado por algunos adversarios y estar dependiendo de opiniones críticas de amigos y correligionarios, ¿sería Brahms un compositor absolutamente romántico? ¿Un espíritu libre del género camerístico? ¿Podría más allá de su música mostrar la belleza espiritual implícita en su alma y su persona? Es claro que el compositor estaba convencido de lo que quería, en el sentido de llevar al más alto ideal la tradición clásica alemana; eso era, como se ha visto, un propósito y una responsabilidad; sin embargo, este reto, tras la sombra de varios gigantes, parece también haber contribuido a ese aparente temor a la hora de enfrentar ciertas frustraciones en las audiciones privadas o públicas de sus obras, o al mostrar sus apuntes musicales a sus colegas. Fue así que constantemente estuvo revisando y rehaciendo partituras hasta encontrar la perfección académica de sus propuestas musicales: estructura, forma y contenido, tendrían cabida en una simbiosis única. En tal sentido, es cierto que "Brahms, al insistir en la renovación de la forma clásica por antonomasia -la sonata-, fue entonces un músico a contracorriente" (Reverter, 1997, s./p.).

En la primera mitad del siglo XX, y durante mucho tiempo, Brahms fue etiquetado como el compositor del Clasicismo-romántico (conservador). Es evidente que su determinación -por unos, criticada y por otros, aplaudida- de utilizar la técnica y las tradiciones de la forma clásica lo ubicaron en un esquema nada revolucionario, en comparación con sus contemporáneos (Wagner, Liszt). 
En el centenario de su natalicio, el compositor Arnold Schoenberg (1874-1951) antes de partir de Alemania, dictó una conferencia que, posteriormente, en 1950, en Estados Unidos, sería publicada como capítulo en su libro El estilo y la idea (Style and Idea), bajo el título "Brahms el progresivo" (Brahms the progressive), cuyo propósito sería demostrar que éste fue un innovador del lenguaje musical y un progresista. Suñén (2010) interpreta que:

El ejemplo, pues, de articulación entre la idea y el estilo es Brahms y el medio para conseguirlo, el sistema más o menos explícito, la fórmula que no es tal de puro lógica y de puro natural, es la variación en desarrollo [...] que, para Schoenberg, que también la utiliza, caracteriza muy especialmente la música de aquel. (p. 10).

El musicólogo, esteta y filósofo, Gabriel Menéndez Torrellas hace referencia a esta técnica:

El método consistía en presentar un tema muy característico y extraer acto seguido de él distintos fragmentos o motivos, que luego volverán a aparecer, en diversas configuraciones, a lo largo de todo el movimiento. Cada una de estas variaciones emerge como una variación del tema, pero estas distintas variaciones, a su vez, no se yuxtaponen unas detrás de otras (como sucedía en el tradicional tema con variaciones, que hemos visto en Haydn, Beethoven o Schubert), sino que siguen también un desarrollo a la vez lógico y afectivo: de ahí entwickelnde Variation, que traducimos como «variación en desarrollo». (2018, p. 166)

En la música de Brahms esta variación se realiza de manera constante y transformadora, lo que implica ciertos resultados que el investigador resume $y$ enumera como características estilísticas que definen la música (sobre todo camerística) del compositor:

- Un movimiento completo nace, por lo general, de un único tema, caracterizado por una constelación de intervalos y un patrón rítmico y melódico.

- La idea inicial se presenta siempre de forma diferente, es un proceso que conduce a nuevas configuraciones.
- Todo lo que se escucha es a la vez constitutivo de la forma y puede experimentar una transformación.

- No hay pasajes de transición, pues todo es desarrollo.

- La reexposición no es una mera repetición de los temas o las ideas iniciales, sino que es única, individual y diversa.

- La coda no es una parte libre que pone el punto final, sino la suma del contenido afectivo y emocional de todo el proceso que la precede. (Menéndez, 2018, p. 167)

Como se puede apreciar, a partir de la forma sonata, el material, las ideas, los motivos, el ritmo, la armonía, el contrapunto, y todos los componentes constitutivos de la composición musical son dominados y de alguna forma renovados, lo que lleva la música alemana a su más alto grado de expresión -dentro del géneroen el romanticismo. A lo anterior Schoenberg agrega como características innovadoras:

...la independencia de los instrumentos en la música de cámara, la casi anulación de la tonalidad fija de algunas piezas, la unidad estructural lograda por el hecho de extraer la mayor parte de una composición de unas pocas ideas básicas, [y] la asimetría métrica... (Kramer, 1993, p. 192)

Para el poeta, escritor y periodista musical Luis Suñén, el reconocimiento de Schoenberg hacia Brahms es la afirmación de la continuidad de una inteligencia en el discurso musical; en otras palabras, es la visión del futuro. A pesar de las diferencias que pudieran apreciarse entre la ruptura y/o la continuidad de la música hacia las vanguardias artísticas del siglo XX, estos principios que Schoenberg descubrió en la música de Brahms, -y que permitieron el cambio en la visión de progreso hacia el compositor-, son los que lo conducirían hacia su invención: la técnica del dodecafonismo como elemento de la vanguardia en la nueva música, la que tantos cambios alentara en el lenguaje musical del siglo XX y hasta la actualidad. Este reconocimiento Suñén (2010) lo justifica así: 
Hay, lo hemos dicho, un componente moral, el aprovechamiento de una lección frente al arte y la vida que el mayor -un Brahms solitario, como una suerte de peñasco que resistiera todas las tormentas- ofrece al más joven desde la última vuelta del camino vital, pero también desde algunas de sus obras tempranas. Probablemente no intuía Schoenberg cuando emprendió este camino de admiración lo que sí vio en 1933 cuando dio su famosa conferencia, es decir, que su camino revolucionario nacía de ahí y él tendría la honradez de reconocerlo mientras acuñaba alguna explicación -la variación progresiva, la prosa musical- para lo que, a partir de esa revelación, ya no sería lo mismo. (pp. 13 y 14).

Muy a tono con A. Schoenberg, Th. W. Adorno (1903-1969) escribió el artículo "Brahms en la actualidad" (1934), y haciendo una revisión de la nueva música, reconocería la trascendencia de Brahms como enlace para la obra de compositores alemanes posteriores, como M. Reger (1873-1916) y P. Hindemith (1895-1963). El filósofo alemán ejemplifica que en Reger, reconocido como "el auténtico eslabón entre el Posclasicismo, el Posromanticismo y la nueva música",

la recuperación de la música absoluta en el marco de la sonata camerística, de la escritura pianística en «acordes» pero más profundamente la técnica de la descomposición en motivos de la unidad de los temas, su transformación mediante el principio del desarrollo por doquier operante y, sobre todo, el estilo de la polifonía armónica son inconcebibles sin Brahms; incluso el fruto más radical de Reger, la «pros» musical gracias a la relajación métrica, está en deuda con las dilataciones y acortamientos brahmsianos. (Adorno, 2011, pp. 208 y 209)

Está claro que, a pesar de las disímiles perspectivas de desarrollo musical, Brahms escogió su propio y único camino. A diferencia de otros compositores, se concentró en lograr la culminación de la maestría clásica, perpetuando la continuidad romántica (Beethoven, Schubert y Schumann), para lo que seleccionó determinados géneros más bien vinculados a las formas tradicionales de la música instrumental, como la sonata, el concierto o la sinfonía, además del lied (música vocal). Trabajó, también, la música coral, instrumental, y las pequeñas formas.
En cuanto a la música de cámara, realizó importantes aportaciones. Si bien sus sinfonías, las obras para piano y sus conciertos son de alta estima en el entorno solístico, igualmente su música de cámara constituye repertorio obligado para los intérpretes consagrados.

\section{La música de cámara en Brahms y sus aportes al desarrollo de la viola}

El espíritu camerístico estuvo siempre presente en la obra general del Brahms compositor: el carácter intimista, el lirismo romántico, las temáticas inspiradoras de la naturaleza (gustaba de salir a componer fuera de la ciudad), de la belleza de la vida, el amor, sus componentes y creaciones (poesía), y el exquisito dominio de los clásicos, más la obra de sus antecesores directos -Schubert, Mendelssohn, Schumannfueron decisivo para su trabajo que comenzó y terminó justo con obras para este género (Trío para piano y cuerdas en Si mayor Op. 8 de 1854 con una nueva versión en 1891, y Sonatas para clarinete y piano Op. $120 n 1$ y $n 2$ (1895). Esto es algo significativo, pues prácticamente Brahms se convierte en un solitario en el momento en que Alemania resiste esta música, predominando la composición de ópera y el poema sinfónico. Según Menéndez (2018):

Brahms no sólo surge al rescate del género; también procuró un equilibrio perfecto entre la función social que la música de cámara burguesa había tenido desde sus inicios y la aspiración de ésta a convertirse en gran música de concierto. Brahms llena el vacío de medio siglo [...] [hasta] el resurgir del cuarteto de cuerda en las primeras obras de la vanguardia del siglo $X X$, representada por Arnold Schönberg y su escuela. (p. 165)

François-René Tranchefort (2005), coordinador de la Guía de la música de cámara, ofrece un análisis de las partituras y las circunstancias en que fueron creadas las obras de cámara de Brahms. Llama la atención en él y otros investigadores, el hecho de que sólo en un período tardío Brahms aborda la forma clásica del cuarteto, a partir de los $O p 51$, y que, de la gran tradición sólo hiciera tres, lo que (al igual que la sinfonía, de las que compuso cuatro) se ha identificado como un particular respeto a la imagen del genio de Beethoven. 
Además de los cuartetos de cuerdas y las sonatas para clarinete (o viola) y piano mencionadas, Brahms compuso otras obras que incluyen o pueden incluir la viola en sustitución de otro instrumento y que son: otros tres cuartetos con piano, un quinteto con piano, un quinteto con clarinete, un trío con clarinete, dos quintetos de cuerdas, y dos sextetos de cuerdas. Su creación original (sin viola) también incluye: tres sonatas para violín y piano, dos sonatas para violonchelo y piano, tres tríos con piano y un trío con trompa.

La música de cámara en Brahms se puede apreciar en tres dimensiones de acuerdo a la cantidad de músicos que integran los ensambles. Esto a su vez representa un tratamiento desde lo más íntimo y personalizado hasta lo proyectado en el sentido sinfónico. Los grupos más pequeños (dúos y tríos) tienden a realizar propuestas más intimistas, de mayor delicadeza, mientras que los quintetos y sextetos, son más como pequeñas sinfonías por la presencia sólida y llena. Los cuartetos constituyen una categoría intermedia $\mathrm{y}$, para ese entonces son ya agrupaciones muy coherentes en el tratamiento particular de cada instrumento y lo compacto del conjunto. (Desde Haydn se considera que los cuartetos de cuerdas son como los talleres preparativos para las creaciones sinfónicas, lo cual se percibe también en Beethoven).

En las obras de cámara de Brahms puede apreciarse desde los primeros opus (Sexteto $n 1$ $o p$ 18) el interés por la viola la cual recibe un tratamiento igualitario respecto al violín y al violonchelo. En muchos casos la viola asume la responsabilidad de los solos, el canto o el contra canto con otro instrumento y no sólo una función armónica. Esto tiene que ver con la idea o propuesta temática, lo que permite destacar algo en particular, ya sea, al inicio de un movimiento determinando el carácter de un tema, o en secuencias de réplicas de motivos, ideas o fragmentos de estas, todo lo cual está perfectamente justificado en la técnica composicional.
Lo interesante en las partituras brahmsianas para el intérprete de la viola es la libertad técnico expresiva en el tratamiento del instrumento por parte del compositor, lo que le permite una participación más activa y creativa en el ensamble. No hay dudas que este lenguaje expresivo es consecuencia de la concepción romántica. Dentro de estas características está la atención detallada a la finura de la emoción en todas sus variantes, contradicciones y expresiones, todo lo cual impulsó la búsqueda de timbres y sonoridades más particulares que podían abrir campos nuevos no explorados hasta ese momento.

Los tres cuartetos para piano y cuerdas, $n 1$, en sol menor Op. 25, n 2, en la mayor Op. 26, y n 3, en do menor Op. 60, fueron compuestos para piano, violín, viola y violonchelo. Los dos primeros (de juventud) están editados desde 1863 y el tercero en 1875 . Aunque se ha dicho que Brahms componía para ensambles inusitados o no característicos para la época (Reverter, 1997) ciertamente este formato había sido utilizado ya por Mozart, quien no sólo fue una influencia determinante en la formación brahmsiana, sino que además se ocupó de dar un tratamiento característico a la viola dentro de estos ensambles, por lo que, de alguna manera, sí se puede observar, desde entonces, un antecedente en algunas propuestas de las consideradas no comunes.

$\mathrm{Al}$ ser Brahms un excelente pianista (al igual que Clara) utiliza este instrumento para su concepción base en muchos de sus ensambles. En el campo que nos ocupa, se puede incluso comparar los cuartetos de piano de Mozart con los de Brahms, por la forma de utilizar la viola, ya que uno y otro están explotando las capacidades del instrumento con suficiente igualdad, (obviamente cada quien en su lenguaje). Pero en los cuartetos de Brahms se siente más el rol de la viola como núcleo del empaste sonoro entre el violín y el violonchelo, lo que hace que el conjunto de las cuerdas sea más competente en el ensamble con el piano, garantizando su firmeza y homogeneidad. Aunque ambos compositores asumen (mediante la asignación de temas, motivos o réplicas) la capacidad expresiva del instrumento, Brahms integra más las voces en su interior y logra un lenguaje más compacto y macizo del conjunto, mientras que, en Mozart, puede observarse todavía la delimitación en el tratamiento de cada voz. 
El tercer cuarteto $O p$. 60 (1875) fue estrenado en 1876 con Brahms interpretando la parte de piano. Su presencia, a más de una década posterior a los $O p$. 25, muestra la consistencia y madurez de un compositor aún con mucho que aportar a la música. "De los tres cuartetos con piano, éste no es solamente el más bello: obra-confesión, sin duda, es también el más libre, evadiéndose muchas veces de los rigores clásicos y solamente guiado por la inspiración del momento." (Tranchefort, 2005, p. 268).

El Quinteto para piano y cuerdas en fa menor Op. 34 (1865) fue estructurado inicialmente para dos violines, una viola y dos cellos; posteriormente Brahms realizó una versión de sonata para dos pianos y, finalmente, quedó como quinteto para piano, dos violines, viola y chelo. Dada su naturaleza, es una obra totalmente experimentada.

Los cuartetos de cuerdas $n 1$, en do menor Op. 51, y $n 2$ en la menor Op. 51, fueron realizados en 1873, mientras que el tercero $n 3$, en si bemol mayor Op. 67 en 1876, casi a la vez que el tercer cuarteto con piano. Luego de un laborioso trabajo de búsqueda en el abordaje del género, donde el compositor se aboca a la perfección y la innovación (lo que Schoenberg reconoce como progreso), Brahms se erige como "«la herencia consciente de la gran tradición de ambición profunda del género» después de Beethoven y Schubert" (Menéndez, 2018, p. 167).

Respecto a los Quintetos de cuerdas n 1, en fa mayor Op. 88 (1883), y $n 2$ en sol mayor Op. 111 (1891), estas son obras particularmente importantes para la viola, pues Brahms escribió (como Mozart) para una dotación de dos violines, dos violas y un violonchelo. Aunque son muy famosos los que le antecedieron de dos chelos -Boccherini, Schubert-, desde finales del siglo XVIII, Michael Haydn (1737-1806) y el propio Boccherini usaban ya el formato con dos violas (Guibert, 2003, p. 9), que también empleó Mendelssohn. Respecto al primero, Brahms expresó a su editor Simrock: "«Puedo decirle que jamás he escuchado una obra mía tan bella»" (Tranchefort, 2005, p. 278). Aunque no fuera bien acogido en Alemania, y sí en el extranjero, el segundo quinteto $O p .111$ fue la última obra que Brahms pensó realizar antes de retirarse.
Tenemos noticia también sobre la poco entusiasta acogida que los íntimos de Brahms Joachim el primero- dispensaron a la nueva partitura: sus reproches se dirigieron especialmente hacia la escritura, que juzgaron complicada, y en particular, parece ser a la importancia que el compositor había dado a la primera viola, instrumento, ciertamente, que gozaba de su predilección. [...] Hanslick fue de muy otra opinión. [...]: «Por lo que se refiere al sentimiento y a la materia musical, el Quinteto tiene los mismos caracteres que las últimas obras en las cuales hemos tenido el placer de ponderar su generosa y espléndida solidez de factura, la intensidad expresiva y la admirable concisión de la forma. Brahms parece concentrarse cada vez más y parece encontrarse cada vez mejor, y con una seguridad siempre creciente, en la expresión fuerte y vigorosa de los sentimientos sencillos.» (Tranchefort, 2005, pp. 280 y 281)

En cuanto a los Sextetos de cuerdas $n 1$, en si bemol mayor Op. 18, y $n$ 2, en sol mayor $O p$. 36, el primero de ellos (1862) constituye su segunda obra del género de cámara; el segundo aparecerá cuatro años más tarde. Compuestos para dos violines, dos violas y dos violonchelos, son bastante originales en su formato (también trabajado por Schoenberg y P. I. Tchaikovsky). Ambos prestan especial atención a la primera viola. En el primer sexteto Brahms asigna temas importantes con características particulares que permiten destacar la viola, incluso su timbre gobierna por completo el movimiento de las variaciones. El tema inicial del primer movimiento Allegro ma non troppo, está a cargo de la viola principal y el violonchelo. El segundo, Andante ma moderato, parece ser una marcha que se desarrolla luego en variaciones; el carácter del movimiento está matizado por el color íntimo y noble de la viola.

Cuando Brahms pensó en retirarse, tuvo lugar la creación de obras con clarinete, las cuales revisten una importancia particular en el desarrollo de la viola. Compuso un trío y un quinteto, ambos concebidos en el verano de 1891, en Bad Ischl, y estrenados en 1892. En el caso del Trío para clarinete, violonchelo $y$ piano en la menor $O p$. 114, el hecho de que el compositor contemplara la posibilidad de sustituir el clarinete por la viola indica tanto el reconocimiento a la misma en su potencialidad con relación al joven instrumento de aliento, como su agrado por la viola. 
El trío fue compuesto para clarinete (o viola), violonchelo y piano. En cuanto a factura, la viola y el violonchelo son más afines desde el punto de vista del género de cámara y en el ensamble de la familia de las cuerdas frotadas, pero Brahms había conocido al primer y excelente clarinetista de la corte de Meiningen, su amigo Richard Mühlfeld (1856-1907) y, a través de él, la técnica del clarinete, con lo que realizó sus últimas obras con este instrumento.

Las dos últimas obras que creó fueron las Sonatas para clarinete (o viola) y piano, la primera en fa menor $O p .120 \mathrm{n} 1$, y la segunda, en mi bemol mayor Op. $120 \mathrm{n} 2$. Ambas fueron compuestas en el verano de 1894; luego de las habituales audiciones privadas que se hicieron en Berchtesgaden (residencia de la corte de Meiningen donde trabajaba como primer clarinetista Mühlfeld) y en Frankfurt, en 1895 se estrenaron en Viena y Leipzig. ${ }^{2}$ Sobre ellas, el humanista, y musicólogo francés Claude Rostand expresó: "son composiciones escritas para uno mismo, como las hojas de un diario íntimo". (Reverter, 1997, p. 17).

Brahms realizó la redacción de la parte de la viola a la muerte de su otro gran amigo, el cirujano, mecenas y violista Theodor Billroth (1829-1894). Organizó la parte de viola agregándole algunas dobles notas y unas terminaciones de fraseo diferentes a la parte original del clarinete. Las variantes de interpretación se enriquecen en la versión para viola por el manejo de diferentes formas expresivas, basadas en el cambio de articulaciones y fraseo, sin lastimar la idea principal del estilo. Igualmente, el registro bajo de la viola agrega brillantez, color y riqueza tímbrica, que matizan el lirismo poético, a veces dramático, otras jovial y jubiloso, de los temas. Ambas sonatas se complementan a pesar de sus diferencias; hay en ellas todo tipo de expresiones y emociones que son difíciles de ignorar cuando se las escucha y, de alguna manera, representan el corolario de su vida como compositor e intérprete.
Por mucho tiempo, las sonatas fueron las composiciones más comprometidas en el repertorio escrito hasta el romanticismo. También, desde el punto de vista técnicoexpresivo constituyeron un pilar, un desafío en el desarrollo de la viola, y como tal abrieron puertas a otras composiciones posrománticas y contemporáneas (siglo XX). Pensar en Brahms para los violistas significaba expresamente el reto de poder interpretar estas sonatas.

Hay una obra muy peculiar en la que Brahms emplea la viola con atinada personalidad, y que además no se corresponde con el género instrumental. Nos referimos a las Canciones para contralto, viola y piano op. 91 , terminadas en 1884 y dedicadas a la cantante austriaca Amalia Joachim, quien la interpretó junto a su esposo, el violinista Joachim tocando la viola y el propio Brahms al piano. Según Poniatovsky (1984), en estas piezas el autor no va por el camino tradicional, sino que es una especie de lied, donde "la unión de la voz baja femenina con el timbre de la viola crea un color sorprendentemente especial. Hay que decir que la parte de viola es sumamente importante en este ensamble." (p. 94) La viola sale como un refuerzo del carácter de las piezas del lied, y a la vez es el ligamento o empaste que sostiene la conjunción del piano y voz.

\footnotetext{
${ }^{2}$ Según Reverter (1997), el estreno oficial tuvo lugar en Viena el 8 y el 11 de enero de 1895. Según Tolpygo, en enero de 1895 recibieron su premiere en las fiestas de cumpleaños de Brahms en Leipzig. Según Tranchefort fueron dadas en primera audición pública en Viena, los días 8 y 11 de enero de 1985 -y posteriormente en otras ciudades, entre ellas Leipzig.
} 


\section{Brahms y el desarrollo potencial de la viola: Nuevas lecturas interpretativas}

Hasta este punto, hemos visto cómo algunos ideales del romanticismo provocaron cambios en el comportamiento y el tratamiento estilístico de la composición, lo que favoreció en particular a la viola, en la obra de Brahms. Esto no es una acción aislada, pues muchos factores han contribuido al desarrollo del instrumento: por un lado, el nacimiento de la escuela de la viola, desde más o menos el mismo período, donde ya tocarla constituye una especialización, surgiendo los primeros conservatorios y sus maestros que se dedican formalmente a ser concertistas y pedagogos de la viola. Por otro, las propias necesidades expresivas de la estética romántica, que también se ejemplifica con Wagner, el cual utiliza la viola dentro de la orquesta de una manera particular, a tal grado de exigir una "reconstrucción" del instrumento, con un tamaño mayor, con el fin de lograr sonoridades específicas para la gran orquesta.

El compositor y teórico Jonathan Kramer, en su libro Invitación a la música, señala:

"Los conciertos para violín de Tchaikovsky, Brahms y Beethoven tienen ciertas cosas en común. Cada uno de ellos fue considerado prácticamente inejecutable cuando era nuevo. Cada uno de ellos actualmente forma parte del repertorio normal de prácticamente todo violinista." (1993, p. 185). Esto ejemplifica lo que justamente pasa con el desarrollo potencial de la viola; es decir, lo que hasta este momento era inimaginable, tanto por la falta de intérpretes como de repertorio, comienza a ser natural a partir del romanticismo por el logro de independencia de las voces interiores y del tratamiento de los compositores hacia las mismas, y particularmente por Brahms, y el contenido de su producción en el género de cámara.

Es sabido que históricamente la viola no tiene -por su función en el conjunto- un amplio catálogo de obras, pero por tradición, partiendo, por ejemplo, del compositor francés M. Marais (1656-1728), en la corte de Luis XIV, los mismos compositores ofrecían la posibilidad de interpretar algunas obras con diferentes variantes de instrumentación (flauta o violín, etc.).
El desarrollo humanístico y artístico provocó cambios, y ya para el período comprendido como romanticismo, fue muy importante el enfoque en el color del instrumento. No sería muy convencional la ocurrencia de tocar el concierto de Dvorak de violonchelo en el clarinete, la viola o la flauta; igualmente interpretar el concierto de Brahms para violín con otro instrumento solista. Lo interesante es que, curiosamente, el diapasón de la viola y el clarinete son parecidos (aunque técnicamente, la escritura brahmsiana de acordes arpegiados en una amplia tesitura de octavas requería de un buen intérprete con suficiente habilidad técnica en la viola).

Quizás ello estimuló a Brahms a ofrecer esas variantes. Obviamente, las características de estos dos instrumentos son muy diferentes, por lo que el enfoque de las interpretaciones es también distinto.

El fenómeno actual es que la viola ha alcanzado tal grado de desarrollo a partir de entonces que los concertistas modernos han desafiado incluso al propio Brahms. Ciertamente, y como toda novedad, no todos los críticos son partidarios de los retos que pueden justificarse desde muchos puntos de vista $\mathrm{y}$ tampoco todos los intérpretes ofrecen variantes plenamente convincentes por el manejo individual de la técnica o la capacidad de expresión tímbrico-sonora; sin embargo, algunas obras no concebidas por Brahms para la viola hoy son interpretadas de esta manera.

Tal es el caso del Quinteto para clarinete y cuerdas en si menor Op. 115, en el que el clarinete es armónicamente respaldado en el conjunto con un cuarteto de cuerdas; actualmente se interpreta también con una viola principal sustituyendo al clarinete y un cuarteto de cuerdas. De emotiva belleza espiritual y una grandeza comprimida, el quinteto tiene una construcción arquitectónica monumental, mientras que en su interior los enlaces son magistralmente desarrollados con toda la libertad, asimetría, la lógica del entrelazamiento de voces y la perfección característica del compositor. Una interpretación no convincente de esta y otras obras brahmsianas puede provocar un gran vacío en el contexto sonoro del auditorio. 
Esta gigantesca obra ha sido consagrada por la interpretación del violista ruso Yuri Bashmet (n. 1953), siendo considerada un hito en la historia de la interpretación violística del siglo XX. Este intérprete realizó la grabación en $\mathrm{CD}$ de la variante de viola con cuarteto, pero también hizo un arreglo para viola y orquesta de cámara que implica la sensibilidad de la orquesta. En esta última versión hay dos aspectos a considerar: uno es que en el original el color del clarinete siempre sobresale en comparación con las cuerdas, lo que hay que observar cuando en lugar del cuarteto actúa una orquesta de cuerdas; el otro, es el cambio de tonalidad. En todas las partes que hemos visto de Brahms no hay cambio de tonalidad en lo referente a las partes con la viola.

Este es un detalle importante, pues Brahms, al igual que Bach, sentía el color de la tonalidad, y esta empatía se encuentra en estas obras que se tocan igualmente con viola o con clarinete.

Otra composición importante, a la vez que rara y particular, es el Trío para piano, violín y trompa, en mi bemol mayor Op. 40. Inspirada en la naturaleza, no es raro que el compositor se acerque lo más posible a ella. Aquí Brahms emplea la trompa de caza, lo cual “...vivifica las sonoridades poéticas y maravillosamente evocadoras: en efecto, son estas cualidades «colorísticas» del instrumento las que Brahms quiso resaltar, rechazando por completo el papel de primer plano, la elocuencia ostentosa de un solista." (Tranchefort, 2005, p. 263). No es de extrañar que entre las variantes interpretativas actuales se sustituya la parte de la trompa por una viola; no obstante, lo extraordinario del intérprete violista es (como se ha hecho), cuando éste sustituye al violín, en cuyo caso -y más allá de las dificultades técnicas- los timbres de la viola y el corno provocan encantadoras sonoridades sui generis.

Aunque las obras mencionadas, y, sobre todo, las dos sonatas $O p$. 120, parecieran suficientes, la exquisita música brahmsiana sigue provocando la avidez de los intérpretes violistas. Por ejemplo, en el 2007 se editó la Sonata Op. 108 en re menor para viola y piano, arreglada y editada por André J. Roy, que se corresponde a la tercera Sonata para violín y piano (1889) en la misma tonalidad. (Roy, 2020).

\section{Conclusiones}

Los cambios estilísticos, el uso de un lenguaje adecuado en cada época, va aparejado a las necesidades expresivas y del quehacer artístico $y$, por tanto, impacta de manera directa en el proceso de perfeccionamiento y adaptación de los instrumentos musicales, así como de la habilitación técnica de los músicos intérpretes. En el romanticismo, la viola alcanzó un papel importante como consecuencia de su potencial tímbrico-sonoro y expresivo, lo cual fue explotado por el compositor Johannes Brahms, particularmente en su creación para los géneros de la música de cámara.

Definitivamente, Brahms fue un estandarte del romanticismo musical, que alcanzó fama y reconocimiento. En su evolución, conoció tanto el éxito como el fracaso. Fue un compositor con dudas y contradicciones propias de la convulsa historia de la música de la segunda mitad del siglo XIX, de las innovaciones y las tradiciones, de las corrientes artísticas musicales y de los procesos socioculturales acaecidos en Europa.

El desarrollo alcanzado por la viola, a partir de la visión brahmsiana, ha despertado el interés de los intérpretes actuales, que han superado con creces las expectativas soñadas, absorbiendo de cada composición todo aquello que pueda aportar al desarrollo potencial tanto del instrumentista como del instrumento.

\section{Referencias}

Adorno, Th. W. (2011). Brahms en la actualidad. Escritos musicales V. Madrid: Akal.

Ars classical (2019). Introduction à la musique romantique. Recuperado de: http://www.arsclassical.com/m-romantique-intro.html.

Consultado: 27 de diciembre de 2020.

Dobrojotov, B. (1962). Conciertos Brandeburgos de J. S. Bach. Moscú: Música.

Domínguez, J. (2009). Lo romántico y el romanticismo en Schlegel, Hegel y Heine. Un debate de cultura política sobre el arte y su tiempo, Revista de Estudios Sociales (34). Bogotá: Universidad de los Andes, pp. 46-58. 
Guibert, A. (2003). Ciclo Quintetos de Mozart, Mendelssohn y Brahms (Introducción general). Madrid: Fundación Juan March.

Hegel, W. F. (1989). Lecciones sobre la estética. Madrid: Akal.

Kramer, J. (1993). Invitación a la música. Buenos Aires: Julio Vergara Editor.

Menéndez, G. (2018). Historia del Cuarteto de Cuerdas. Madrid: Akal.

Plantinga, León (2015). La música romántica. Madrid: Akal.

Poniatovsky, S. (1984). Historia del arte violístico. Moscú: Música, Moscú.

Reverter, A. (1997). Ciclo Brahms, Música de cámara (Introducción general y Notas). Madrid: Fundación Juan March.

Roy, A. (Ed.) (2020). Sonata in D minor, $O p$ 108. Recuperado de: https://www.sharmusic.com/SheetMusic/Viola/w-47-Piano/Brahms-Johannes--Sonata-in-D-minor-Op-108---transcribed-forViola-and-Piano-by-Andrea-Roy---EditionsRoyalto.axd. Consultado: 26 de diciembre de 2020.

Safranski, R. (2009). Romanticismo. Una odisea del espíritu alemán. Barcelona: Tusquets.

Suñén, L. (2010). "Brahms, el progresista": un programa de Schonberg (Introducción y Notas). Madrid: Fundación Juan March.

Tolpygo, M., y Mndoyan, K. (1982). Brahms, Sonatas para viola y piano, [Long Play] URSS: Melodía.

Tranchefort, F. R. (2005). Guía de la música de cámara. Madrid: Alianza Editorial. 